\title{
Weak Solutions for Partial Random Hadamard Fractional Integral Equations with Multiple Delays
}

\author{
Saïd Abbas, ${ }^{1}$ Mouffak Benchohra, ${ }^{2}$ Yong Zhou, ${ }^{3,4}$ and Ahmed Alsaedi ${ }^{4}$ \\ ${ }^{1}$ Laboratory of Mathematics, University of Saïda, P.O. Box 138 ENNASR, 20000 Saïda, Algeria \\ ${ }^{2}$ Laboratory of Mathematics, University of Sidi Bel-Abbès, P.O. Box 89, 22000 Sidi Bel-Abbès, Algeria \\ ${ }^{3}$ Faculty of Mathematics and Computational Science, Xiangtan University, Hunan 411105, China \\ ${ }^{4}$ Department of Mathematics, Faculty of Science, King Abdulaziz University, P.O. Box 80203, Jeddah 21589, Saudi Arabia
}

Correspondence should be addressed to Yong Zhou; yzhou@xtu.edu.cn

Received 23 June 2017; Accepted 14 September 2017; Published 15 October 2017

Academic Editor: Jorge E. Macías-Díaz

Copyright (C) 2017 Saïd Abbas et al. This is an open access article distributed under the Creative Commons Attribution License, which permits unrestricted use, distribution, and reproduction in any medium, provided the original work is properly cited.

We present some results concerning the existence of weak solutions for some functional integral equations of Hadamard fractional order with random effects and multiple delays by applying Mönch's and Engl's fixed point theorems associated with the technique of measure of weak noncompactness.

\section{Introduction}

Random differential equations arise in many applications and have been studied in the literature on bounded as well as unbounded internals of the real line for different aspects of the solution. See, for example, [1]. We refer the reader to the monograph [2] and the papers $[1,3]$ and the references therein. There are real-world phenomena with anomalous dynamics such as signals transmissions through strong magnetic fields, atmospheric diffusion of pollution, network traffic, and the effect of speculations on the profitability of stocks in financial markets, where the classical models are not sufficiently good to describe these features. In this case, the theory of fractional differential equations is a good tool for modeling such phenomena. Therefore, the study of the fractional differential equations with random parameters seems to be a natural one. For some fundamental results in the theory of fractional calculus and fractional differential equations, we refer the reader to the monographs of Abbas et al. [4,5], Baleanu et al. [6], and Kilbas et al. [7] and a series of recent research articles [8-12] and the references therein.

The measure of weak noncompactness is introduced by De Blasi [13]. The strong measure of noncompactness was developed first by Banaś and Goebel [14] and subsequently developed and used in many papers; see, for example,
Akhmerov et al. [15], Alvàrez [16], and Guo et al. [17] and the references therein. In [18], the authors considered some existence results by applying the techniques of the measure of noncompactness. Recently, several researchers obtained other results by application of the technique of measure of weak noncompactness; see [5]. Existence of random solutions for functional differential and integral equations has extensively been studied in various papers; see $[19,20]$ and the references therein.

In this paper, we discuss the existence of random solutions for the partial Hadamard fractional integral equation of the following form:

$$
\begin{aligned}
& u(x, y, w) \\
& =\sum_{i=1}^{m} b_{i}(x, y, w) u\left(x-\xi_{i}, y-\mu_{i}, w\right) \\
& \quad+f\left(x, y,{ }^{H} I_{\sigma}^{r} u(x, y, w), u(x, y, w), w\right) ; \\
& \quad(x, y) \in J, w \in \Omega, \\
& u(x, y, w)=\Phi(x, y, w) ; \quad \text { if }(x, y) \in \widetilde{J}, w \in \Omega,
\end{aligned}
$$

where $J:=[1, a] \times[1, b], \widetilde{J}:=[-\xi, a] \times[-\mu, b] \backslash(1, a] \times(1, b]$, $a, b>1, \xi_{i}, \mu_{i} \geq 1(i=1, \ldots, m), \xi=\max _{i=1, \ldots, m}\left\{\xi_{i}\right\}$, 
$\mu=\max _{i=1, \ldots, m}\left\{\mu_{i}\right\}, \sigma=(1,1), r=\left(r_{1}, r_{2}\right) \in(0, \infty) \times(0, \infty)$, $b_{i}: J \times \Omega \rightarrow \mathbb{R}(i=1, \ldots, m)$, and $f: J \times E \times E \times \Omega \rightarrow$ $E$ are given continuous functions, $(\Omega, \mathscr{A}, v)$ is a measurable space, and $E$ is a real (or complex) Banach space with norm $\|\cdot\|_{E}$ and dual $E^{*}$, such that $E$ is the dual of a weakly compactly generated Banach space $X,{ }^{H} I_{\sigma}^{r}$ is the left-sided mixed Hadamard integral of order $r$, and $\Phi: \widetilde{J} \times \Omega \rightarrow E$ is a given continuous and measurable function such that

$$
\begin{gathered}
\Phi(x, 1, w)=\sum_{i=1}^{m} b_{i}(x, 1, w) \Phi\left(x-\xi_{i}, 1-\mu_{i}, w\right) ; \\
x \in[1, a], w \in \Omega, \\
\Phi(1, y, w)=\sum_{i=1}^{m} b_{i}(1, y, w) \Phi\left(1-\xi_{i}, y-\mu_{i}, w\right) ; \\
y \in[1, b], w \in \Omega .
\end{gathered}
$$

\section{Preliminaries}

Let $\mathscr{C}$ be the Banach space of all continuous functions from $[-\xi, a] \times[-\mu, b]$ into $E$ with the supremum (uniform) norm $\|\cdot\|_{\infty}$. By $L^{\infty}(\Omega, \nu)$, we denote the Banach space of measurable functions $u: \Omega \rightarrow C$ which are essentially bounded equipped with the norm

$$
\begin{aligned}
\|u\|_{L^{\infty}} & :=\sup _{w \in \Omega} \operatorname{ess}\|u(w)\|_{C} \\
& =\inf \left\{c>0:\|u(w)\|_{C} \leq c \nu \text {-a.e. in } \Omega\right\} .
\end{aligned}
$$

Denote by $(E, w)=\left(E, \sigma\left(E, E^{*}\right)\right)$ the Banach space $E$ with its weak topology.

Definition 1. A Banach space $X$ is called weakly compactly generated (WCG, in short) if it contains a weakly compact set whose linear span is dense in $X$.

Definition 2. A function $h: E \rightarrow E$ is said to be weakly sequentially continuous if $h$ takes each weakly convergent sequence in $E$ to a weakly convergent sequence in $E$ (i.e., for any $\left(u_{n}\right)$ in $E$ with $u_{n} \rightarrow u$ in $(E, w)$, one has $h\left(u_{n}\right) \rightarrow h(u)$ in $(E, w))$.

Definition 3 (see [21]). The function $u: J \rightarrow E$ is said to be Pettis integrable on $J$ if and only if there is an element $u_{j} \in E$ corresponding to each $j \subset J$ such that $\phi\left(u_{j}\right)=$ $\iint_{j} \phi(u(s, t)) d t d s$ for all $\phi \in E^{*}$, where the integral on the right-hand side is assumed to exist in the sense of Lebesgue (by definition, $u_{j}=\iint_{j} u(s, t) d t d s$ ).

Let $P(J, E)$ be the space of all $E$-valued Pettis integrable functions on $J$, and let $L^{1}(J, \mathbb{R})$ be the Banach space of Lebesgue measurable functions $u: J \rightarrow \mathbb{R}$. Define the class $P_{1}(J, E)$ by

$$
\begin{aligned}
& P_{1}(J, E)=\{u \in P(J, E): \varphi(u) \\
& \left.\quad \in L^{1}(J, \mathbb{R}) ; \text { for every } \varphi \in E^{*}\right\} .
\end{aligned}
$$

The space $P_{1}(J, E)$ is normed by

$$
\|u\|_{P_{1}}=\sup _{\varphi \in E^{*},\|\varphi\| \leq 1} \int_{1}^{a} \int_{1}^{b}|\varphi(u(x, y))| d \lambda(x, y),
$$

where $\lambda$ stands for a Lebesgue measure on $J$.

The following result is due to Pettis (see [21], Theorem 3.4 and Corollary 3.41).

Proposition 4 (see [21]). If $u \in P_{1}(J, E)$ and $h$ is a measurable and essentially bounded E-valued function, then uh $\in P_{1}(J, E)$.

For all what follows, the sign " $\int$ " denotes the Pettis integral.

Let us recall the definitions of Pettis integral and Hadamard integral of fractional order.

Definition 5 (see [7]). The left-sided mixed Pettis Hadamard integral of order $q>0$, for a function $g \in P_{1}([1, a], E)$, is defined as

$$
\left({ }^{H} I_{1}^{r} g\right)(x)=\frac{1}{\Gamma(q)} \int_{1}^{x}\left(\ln \frac{x}{s}\right)^{q-1} \frac{g(s)}{s} d s .
$$

Remark 6. Let $g \in P_{1}([1, a], E)$. For every $\varphi \in E^{*}$, one has

$$
\varphi\left({ }^{H} I_{1}^{r} g\right)(x)=\left({ }^{H} I_{1}^{r} \varphi g\right)(x) ; \quad \text { for a.e. } x \in[1, a] .
$$

Definition 7. Let $r_{1}, r_{2} \geq 0, \sigma=(1,1)$, and $r=\left(r_{1}, r_{2}\right)$. For $w \in P_{1}(J, E)$, define the left-sided mixed Pettis Hadamard partial fractional integral of order $r$ by the expression

$$
\begin{aligned}
& \left({ }^{H} I_{\sigma}^{r} w\right)(x, y)=\frac{1}{\Gamma\left(r_{1}\right) \Gamma\left(r_{2}\right)} \\
& \cdot \int_{1}^{x} \int_{1}^{y}\left(\ln \frac{x}{s}\right)^{r_{1}-1}\left(\ln \frac{y}{t}\right)^{r_{2}-1} \frac{w(s, t)}{s t} d t d s .
\end{aligned}
$$

Let $\beta_{E}$ be the $\sigma$-algebra of Borel subsets of $E$. A mapping $v: \Omega \rightarrow E$ is said to be measurable if, for any $B \in \beta_{E}$, one has

$$
v^{-1}(B)=\{w \in \Omega: v(w) \in B\} \in \mathscr{A} .
$$

To define integrals of sample paths of random process, it is necessary to define a jointly measurable map.

Definition 8. A function $T: \Omega \times E \rightarrow E$ is called jointly measurable if, for any $B \in \beta_{E}$, one has

$$
T^{-1}(B):=\{(w, v) \in \Omega \times E: T(w, v) \in B\} \in \mathscr{A} \times \beta_{E},
$$

where $\mathscr{A} \times \beta_{E}$ is the direct product of the $\sigma$-algebras $\mathscr{A}$ and $\beta_{E}$, those defined in $\Omega$ and $E$, respectively.

Lemma 9 (see [22]). A function $T: \Omega \times E \rightarrow E$ is jointly measurable if $T(\cdot, u)$ is measurable for all $u \in E$ and $T(w, \cdot)$ is continuous for all $w \in \Omega$.

Definition 10. A function $f: J \times E \times E \times \Omega \rightarrow E$ is called random Carathéodory if the following conditions are satisfied: 
(i) The map $(x, y, w) \rightarrow f(x, y, u, v, w)$ is jointly measurable for all $u, v \in E$.

(ii) The map $(u, v) \rightarrow f(x, y, u, v, w)$ is continuous for all $(x, y) \in J$ and $w \in \Omega$.

Let $T: \Omega \times E \rightarrow E$ be a mapping. Then $T$ is called a random operator if $T(w, u)$ is measurable in $w$ for all $u \in E$ and it is expressed as $T(w) u=T(w, u)$. In this case, we also say that $T(w)$ is a random operator on $E$. A random operator $T(w)$ on $E$ is called continuous (resp., compact, totally bounded, and completely continuous) if $T(w, u)$ is continuous (resp., compact, totally bounded, and completely continuous) in $u$ for all $w \in \Omega$. The details of completely continuous random operators in Banach spaces and their properties appear in Itoh [23].

Definition 11 (see [24]). Let $\mathscr{P}(Y)$ be the family of all nonempty subsets of $Y$ and let $C$ be a mapping from $\Omega$ into $\mathscr{P}(Y)$. A mapping $T:\{(w, y): w \in \Omega, y \in C(w)\} \rightarrow Y$ is called random operator with stochastic domain $C$ if $C$ is measurable (i.e., for all closed $A \subset Y,\{w \in \Omega, C(w) \cap A \neq \emptyset\}$ is measurable) and for all open $D \subset Y$ and all $y \in Y$, $\{w \in \Omega: y \in C(w), T(w, y) \in D\}$ is measurable. $T$ will be called continuous if every $T(w)$ is continuous. For a random operator $T$, a mapping $y: \Omega \rightarrow Y$ is called random (stochastic) fixed point of $T$ if for $P$ - almost all $w \in \Omega$, $y(w) \in C(w)$, and $T(w) y(w)=y(w)$ and for all open $D \subset Y$, $\{w \in \Omega: y(w) \in D\}$ is measurable.

Definition 12 (see [13]). Let $E$ be a Banach space, let $\Omega_{E}$ be the bounded subsets of $E$, and let $B_{1}$ be the unit ball of $E$. De Blasi measure of weak noncompactness is the map $\beta: \Omega_{E} \rightarrow$ $[0, \infty)$ defined by

$$
\beta(X)=\inf \{\epsilon>0:
$$

there exists a weakly compact subset $\Omega$ of $E: X$

$$
\left.\subset \epsilon B_{1}+\Omega\right\} \text {. }
$$

De Blasi measure of weak noncompactness satisfies the following properties:
(a) $A \subset B \Rightarrow \beta(A) \leq \beta(B)$.
(b) $\beta(A)=0 \Leftrightarrow A$ is weakly relatively compact.
(c) $\beta(A \cup B)=\max \{\beta(A), \beta(B)\}$
(d) $\beta\left(\bar{A}^{\omega}\right)=\beta(A)\left(\bar{A}^{\omega}\right.$ denotes the weak closure of $\left.A\right)$.
(e) $\beta(A+B) \leq \beta(A)+\beta(B)$.
(f) $\beta(\lambda A)=|\lambda| \beta(A)$.
(g) $\beta(\operatorname{conv}(A))=\beta(A)$.
(h) $\beta\left(\bigcup_{|\lambda| \leq h} \lambda A\right)=h \beta(A)$.

The next result follows directly from the Hahn-Banach theorem.

Proposition 13. Let $E$ be a normed space, and $x_{0} \in E$ with $x_{0} \neq 0$. Then, there exists $\varphi \in E^{*}$ with $\|\varphi\|=1$ and $\varphi\left(x_{0}\right)=$ $\left\|x_{0}\right\|$.
For a given set $V$ of functions $v: J \rightarrow E$, let us denote that

$$
\begin{gathered}
V(x, y)=\{v(x, y): v \in V\} ; \quad(x, y) \in J, \\
V(J)=\{v(x, y): v \in V, \quad(x, y) \in J\} .
\end{gathered}
$$

Lemma 14 (see [17]). Let $H \subset C$ be bounded and equicontinuous. Then the function $(x, y) \rightarrow \beta(H(x, y))$ is continuous on $J$, and

$$
\begin{aligned}
\beta_{C}(H) & =\max _{(x, y) \in J} \beta(H(x, y)), \\
\beta\left(\iint_{J} u(s, t) d t d s\right) & \leq \iint_{J} \beta(H(s, t)) d t d s,
\end{aligned}
$$

where $H(x, y)=\{u(x, y): u \in H\} ;(x, y) \in J$, and $\beta_{C}$ is De Blasi measure of weak noncompactness defined on the bounded sets of $C$.

We will need the following fixed point theorems.

Theorem 15 (see [25]). Let $Q$ be a nonempty, closed, convex, and equicontinuous subset of a metrizable locally convex vector space $C(J, E)$ such that $0 \in Q$. Suppose that $T: Q \rightarrow Q$ is weakly, sequentially continuous. If the implication

$$
\bar{V}=\overline{\operatorname{conv}}(\{0\} \cup T(V)) \Longrightarrow V \text { is relatively weakly compact }
$$

holds for every subset $V \subset Q$, then the operator $T$ has a fixed point.

Theorem 16 (see [24]). Let $C: \Omega \rightarrow 2^{Y}$ be measurable with closed, convex, and solid $C(w)$ (i.e., int $C(w) \neq \emptyset$ ) for all $w \in \Omega$. We assume that there exists measurable $y_{0}: \Omega \rightarrow Y$ with $y_{0} \in$ int $C(w)$ for all $w \in \Omega$. Let $T$ be a continuous random operator with stochastic domain $C$ such that, for every $w \in \Omega,\{y \in$ $C(\omega): T(w) y=y\} \neq \emptyset$. Then $T$ has a stochastic fixed point.

\section{Existence Results}

Let us start by defining what we mean by a random solution of problem (1).

Definition 17. By a random solution of problem (1), we mean a measurable function $u: \Omega \rightarrow C([-\xi, a] \times[-\mu, b])$ that satisfies the integral equation $u(x, y, w)=\sum_{i=1}^{m} b_{i}(x, y, w) u\left(x-\xi_{i}, y-\right.$ $\left.\mu_{i}, w\right)+f\left(x, y,{ }^{H} I_{\sigma}^{r} u(x, y, w), u(x, y, w), w\right)$ on $J \times \Omega$, as well as $u(x, y, w)=\phi(x, y, w)$ on $\widetilde{J} \times \Omega$.

The following hypotheses will be used in the sequel:

$\left(H_{1}\right)$ The functions $w \mapsto b_{i}(x, y, w), i=1, \ldots, m$, are bounded for a.e. $(x, y) \in J$, and $b_{i}(\cdot, \cdot, w) \in L^{\infty}(J, \mathbb{R})$.

$\left(\mathrm{H}_{2}\right)$ The function $f$ is random Carathéodory on $J \times E \times$ $E \times \Omega$ for each $w \in \Omega$.

$\left(H_{3}\right)$ For a.e. $(x, y) \in J$, as well as all $w \in \Omega$, the function $u \rightarrow f\left(x, y,{ }^{H} I_{\sigma} u, u, w\right)$ is weakly, sequentially continuous. 
$\left(H_{4}\right)$ There exist functions $p_{1}, p_{2}, p_{3}: J \times \Omega \rightarrow[0, \infty)$ with $p_{i}(., w) \in L^{\infty}(J,[0, \infty)), i=1,2,3$, for each $w \in \Omega$, such that, for all $\varphi \in E *$, one has

$$
\begin{aligned}
& |\varphi(f(x, y, u, v, w))| \\
& \leq \frac{p_{1}(x, y, w)\|\varphi\|+p_{2}(x, y, w)\|u\|_{E}+p_{3}(x, y, w)\|v\|_{E}}{1+\|\varphi\|},
\end{aligned}
$$

for all $u, v \in E$ and a.e. $(x, y) \in J$.

$\left(H_{5}\right)$ For all $u \in E$, there exists a continuous function $\psi$ : $[0, \infty) \rightarrow[0, \infty)$ with $\psi(0)=0$, such that, for each $\varphi \in E^{*},\left(x_{1}, y_{1}\right),\left(x_{2}, y_{2}\right) \in J$ and any $w \in \Omega$, one has

$$
\begin{aligned}
& \sum_{i=1}^{m} \mid b_{i}\left(x_{1}, y_{1}, w\right) u\left(x_{1}-\xi_{i}, y_{1}-\mu_{i}, w\right)-b_{i}\left(x_{2}, y_{2}, w\right) \\
& \cdot u\left(x_{2}-\xi_{i}, y_{2}-\mu_{i}, w\right)|+| \varphi(f(x \\
& \quad-1, y_{1}, u\left(x_{1}, y_{1}\right), v\left(x_{1}, y_{1}, w\right) \\
& \left.\left.-f\left(x_{2}, y_{2}, u\left(x_{2}, y_{2}\right), v\left(x_{2}, y_{2}\right), w\right)\right)\right) \mid \\
& \quad \leq \frac{\psi\left(\left|x_{1}-x_{2}\right|+\left|y_{1}-y_{2}\right|\right)\|\varphi\|}{1+\|\varphi\|+\|u\|_{E}+\|v\|_{E}} .
\end{aligned}
$$

$\left(H_{6}\right)$ There exists a function $q: J \times \Omega \rightarrow[0, \infty)$ with $q(\cdot, \cdot, w) \in L^{\infty}(J,[0, \infty))$ for each $w \in \Omega$ such that, for any bounded $B \subset E$,

$$
\begin{array}{r}
\alpha\left(f\left(x, y,{ }^{H} I_{\sigma} B, B, w\right)\right) \leq q(x, y, w) \alpha(B), \\
\text { for a.e. }(x, y) \in J,
\end{array}
$$

where ${ }^{H} I_{\sigma} B:=\left\{{ }^{H} I_{\sigma} u(x, y): u(x, y) \in B ;(x, y) \in J\right\}$.

$\left(H_{7}\right)$ There exists a random function $R: \Omega \rightarrow(0, \infty)$ such that

$$
\begin{aligned}
p_{1}^{*}(w) & +\left(m b^{*}+p_{3}^{*}(w)\right) R(w) \\
+ & \frac{p_{2}^{*}(w) R(w)(\log a)^{r_{1}}(\log b)^{r_{2}}}{\Gamma\left(1+r_{1}\right) \Gamma\left(1+r_{2}\right)} \leq R(w),
\end{aligned}
$$

where

$$
\begin{aligned}
b^{*} & =\max _{i=1, \ldots, m}\left\{\operatorname{essup}_{(x, y, w) \in J \times \Omega}\left|b_{i}(x, y)\right|\right\}, \\
p_{i}^{*}(w) & =\underset{(x, y) \in J}{\operatorname{ess} \sup } p_{i}(x, y, w) ; \quad i=1,2,3 .
\end{aligned}
$$

Set

$$
q^{*}=\operatorname{ess}_{(x, y, w) \in J \times \Omega} q(x, y, w) .
$$

Theorem 18. Assume that hypotheses $\left(H_{1}\right)-\left(H_{7}\right)$ hold. If

$$
\ell:=m b^{*}+q^{*}<1,
$$

then problem (1) has a random solution defined on $[-\xi, a] \times$ $[-\mu, b]$.
Proof. Define the operator $N: \Omega \times \mathscr{C} \rightarrow \mathscr{C}$ by

$$
\begin{aligned}
& (N(w) u)(x, y) \\
& =\sum_{i=1}^{m} b_{i}(x, y, w) u\left(x-\xi_{i}, y-\mu_{i}, w\right) \\
& \quad+f\left(x, y,{ }^{H}{ }^{r}{ }_{\sigma}^{r} u(x, y, w), u(x, y, w), w\right) ;
\end{aligned}
$$

$$
(x, y) \in J
$$

$(N(w) u)(x, y)=\Phi(x, y, w) ; \quad(x, y) \in \widetilde{J}$.

The functions $\Phi, b_{i}, i=1, \ldots, m$, are continuous for all $w \in \Omega$. Again, as the function $f$ is continuous on $J, N(w)$ defines a mapping $N: \Omega \times \mathscr{C} \rightarrow \mathscr{C}$. Thus, $u$ is a solution for problem (1) if and only if $u=(N(w)) u$. We shall show that the operator $N$ satisfies all conditions of Theorem 16 . The proof will be given in several steps.

Step $1(N(w)$ is a random operator with stochastic domain on $\mathscr{C})$. Since $f(x, y, u, v, w)$ is random Carathéodory, the map $w \rightarrow f(x, y, u, v, w)$ is measurable in view of Definition 10 . Therefore, the map

$$
\begin{aligned}
w \longmapsto & \sum_{i=1}^{m} b_{i}(x, y, w) u\left(x-\xi_{i}, y-\mu_{i}, w\right) \\
& +f\left(x, y,{ }^{H} I_{\sigma}^{r} u(x, y, w), u(x, y, w), w\right)
\end{aligned}
$$

is measurable. As a result, $N$ is a random operator on $\Omega \times \mathscr{C} \times \mathscr{C}$ into $\mathscr{C}$.

$$
\begin{aligned}
& \text { Let } W: \Omega \rightarrow \mathscr{P}(C) \text { be defined by } \\
& W(w)=\left\{u \in C:\|u\|_{C}\right. \\
& \leq R(w),\left\|u\left(x_{1}, y_{1}, w\right)-u\left(x_{2}, y_{2}, w\right)\right\|_{E} \\
& \left.\leq \psi\left(\left|x_{1}-x_{2}\right|+\left|y_{1}-y_{2}\right|\right)\right\} .
\end{aligned}
$$

Clearly, the subset $W(w)$ is closed, convex, end equicontinuous for all $w \in \Omega$. Then $W$ is measurable by Lemma 17 in [24]. Therefore, $N$ is a random operator with stochastic domain $W$.

Step $2\left(N(w)\right.$ is continuous). Let $\left\{u_{n}\right\}$ be a sequence such that $u_{n} \rightarrow u$ in $\mathscr{C}$. Then, there exists $\phi \in E^{*}$ such that $\left\|\left(N(w) u_{n}\right)(x, y)\right\|_{E}=\phi\left(\left(N(w) u_{n}\right)(x, y)\right)$, and $\|(N(w) u)(x, y)\|_{E}=\phi((N(w) u)(x, y))$.

Thus, for each $(x, y) \in J$ and $w \in \Omega$, one has

$$
\begin{aligned}
& \left\|\left(N(w) u_{n}\right)(x, y)-(N(w) u)(x, y)\right\|_{E} \\
& \quad=\phi\left(\left(N(w) u_{n}\right)(x, y)-(N(w) u)(x, y)\right) \\
& \quad \leq \sum_{i=1}^{m}\left|b_{i}(x, y, w)\right| \| u_{n}\left(x-\xi_{i}, y-\mu_{i}, w\right)-u(x \\
& \left.\quad-\xi_{i}, y-\mu_{i}, w\right) \|_{E} \\
& +\mid \phi\left(f\left(x, y,{ }^{H} I_{\sigma}^{r} u_{n}(x, y, w), u_{n}(x, y, w), w\right)\right. \\
& \left.-f\left(x, y,{ }^{H} I_{\sigma}^{r} u(x, y, w), u(x, y, w), w\right)\right) \mid .
\end{aligned}
$$


Using the Lebesgue Dominated Convergence Theorem, we get

$$
\left\|N(w) u_{n}-N(w) u\right\|_{\infty} \longrightarrow 0 \text { as } n \longrightarrow \infty \text {. }
$$

As a consequence of Steps 1 and 2, we can conclude that $N(w): W(w) \rightarrow N(w)$ is a continuous random operator with stochastic domain $W$, and $N(w)(W(w))$ is bounded.

Step 3 (for every $w \in \Omega,\{u \in W(w): N(w) u=u\} \neq \emptyset$ ). For this, we apply Theorem 15 . The proof will be given in several claims.

Claim $1(N(w)$ maps $W(w)$ into itself). Let $w \in \Omega$ be fixed, and let $u \in W(w),(x, y) \in J$. Assume that $(N(w) u)(x, y) \neq$ 0 . Then there exists $\phi \in E^{*}$ such that $\|(N(w) u)(x, y)\|_{E}=$ $\phi((N(w) u)(x, y))$. Thus, we get

$$
\begin{aligned}
& \|(N(w) u)(x, y)\|_{E} \leq \| \sum_{i=1}^{m} b_{i}(x, y, w) \\
& \cdot u\left(x-\xi_{i}, y-\mu_{i}, w\right) \|_{E} \\
& +\left|\phi\left(f\left(x, y,{ }^{H} I_{\sigma}^{r} u(x, y, w), u(x, y, w), w\right)\right)\right| \\
& \quad \leq \sum_{i=1}^{m}\left|b_{i}(x, y, w)\right|\left\|u\left(x-\xi_{i}, y-\mu_{i}, w\right)\right\|_{E} \\
& +p_{1}(x, y, w)+p_{2}(x, y, w)\left\|^{H} I_{\sigma}^{r} u(x, y, w)\right\|_{E} \\
& +p_{3}(x, y, w)\|u(x, y, w)\|_{E} \leq m b^{*}\|u\|_{\infty}+p_{1}^{*}(w) \\
& +\frac{p_{2}^{*}(w)}{\Gamma\left(r_{1}\right) \Gamma\left(r_{2}\right)} \int_{1}^{x} \int_{1}^{y}\left(\log \frac{x}{s}\right)^{r_{1}-1}\left(\log \frac{y}{t}\right)^{r_{2}-1} \\
& +\|u(s, t, w)\|_{E} d t d s+p_{3}^{*}(w) R(w) \leq p_{1}^{*}(w) \\
& +\left(m b^{*}+p_{3}^{*}(w)\right) R(w) \\
& +\frac{p_{2}^{*}(w) R(w)(\log a)^{r_{1}}(\log b)^{r_{2}}}{\Gamma\left(1+r_{1}\right) \Gamma\left(1+r_{2}\right)} \leq R(w) .
\end{aligned}
$$

Next, for any fixed $w \in \Omega$, let $\left(x_{1}, y_{1}\right),\left(x_{2}, y_{2}\right) \in J$ such that $x_{1}<x_{2}$ and $y_{1}<y_{2}$, and let $u \in W(w)$, where $(N(w) u)\left(x_{1}, y_{1}\right)-(N(w) u)\left(x_{2}, y_{2}\right) \neq 0$. Then there exists $\phi \in E^{*}$ such that $\left\|(N(w) u)\left(x_{1}, y_{1}\right)-(N(w) u)\left(x_{2}, y_{2}\right)\right\|_{E}=$ $\phi\left((N(w) u)\left(x_{1}, y_{1}\right)-(N(w) u)\left(x_{2}, y_{2}\right)\right)$ and $\|\varphi\|=1$. Thus, one has

$$
\begin{aligned}
& \left\|(N(w) u)\left(x_{2}, y_{2}\right)-(N(w) u)\left(x_{1}, y_{1}\right)\right\|_{E} \\
& \quad=\phi\left((N(w) u)\left(x_{2}, y_{2}\right)-(N(w) u)\left(x_{1}, y_{1}\right)\right) \\
& \quad \leq \psi\left(\left|x_{1}-x_{2}\right|+\left|y_{1}-y_{2}\right|\right) .
\end{aligned}
$$

Hence $N(W(w)) \subset W(w)$. Therefore, $N(w): W(w) \rightarrow N(w)$ maps $W(w)$ into itself.

Claim $2\left(N(w)\right.$ is weakly, sequentially continuous). Let $\left(u_{n}\right)$ be a sequence in $W(w)$ and let $\left(u_{n}(x, y, w)\right) \rightarrow u(x, y, w)$ in $(E, \omega)$ for any $w \in \Omega$, and each $(x, y) \in J$. Fix $(x, y)$ $\epsilon J$, and since $f$ satisfies assumption $\left(H_{6}\right)$, one has that $f\left(x, y,{ }^{H} I_{\sigma} u_{n}(x, y, w), u_{n}(x, y, w), w\right)$ converges weakly uniformly to

$$
f\left(x, y,{ }^{H} I_{\sigma} u(x, y, w), u(x, y, w), w\right) .
$$

Hence, the Lebesgue Dominated Convergence Theorem for Pettis integral implies that $\left(N u_{n}\right)(x, y, w)$ converges weakly uniformly to $(N(w) u)(x, y)$ in $(E, \omega)$. We do it for any $w \in \Omega$, and each $(x, y) \in J$, so $N(w)\left(u_{n}\right) \rightarrow N(w)(u)$. Then $N: W(w) \rightarrow W(w)$ is weakly, sequentially continuous.

Claim 3 (implication (14) holds). Let $V$ be a subset of $W(w)$ such that $\bar{V}=\overline{\operatorname{conv}}(N(w)(V) \cup\{0\})$. Obviously, $V(x, y, w) \subset \overline{\operatorname{conv}}((N(w) V)(x, y) \cup\{0\})$. Further, as $V$ is bounded and equicontinuous, by Lemma 3 in [26], the function $(x, y, w) \rightarrow u(x, y, w)=\beta(V(x, y, w))$ is continuous on $J \times \Omega$. Since the function $\mu$ is continuous on $J \times \Omega$, the set $\{\mu(x, y, w) ;(x, y) \in J, w \in \Omega\} \subset E$ is compact. From Lemma 14 and the properties of the measure $\beta$, for any $w \in \Omega$, as well as each $(x, y) \in J$, one has

$$
\begin{aligned}
& v(x, y, w) \leq \beta((N(w) V)(x, y) \cup\{0\}) \\
& \quad \leq \beta((N(w) V)(x, y)) \\
& \quad=\beta\left(\sum_{i=1}^{m} b_{i}(x, y, w) u\left(x-\xi_{i}, y-\mu_{i}, w\right)\right. \\
& \left.+f\left(x, y,{ }^{H} I_{\sigma}^{r} u(x, y, w), u(x, y, w), w\right)\right) \\
& \quad \leq \sum_{i=1}^{m}\left|b_{i}(x, y, w)\right| \beta(V(x, y, w))+q(x, y, w) \\
& \cdot \beta(V(x, y, w)) \leq m b^{*} v(x, y, w)+q^{*} v(x, y, w) \\
& \leq\left(m b^{*}+q^{*}\right)\|v\|_{C} .
\end{aligned}
$$

Thus,

$$
\|v\|_{C} \leq \ell\|v\|_{C}
$$

From (21), we get $\|v\|_{C}=0$, that is; $v(x, y, w)=$ $\beta(V(x, y, w))=0$, for any $w \in \Omega$, and each $(x, y) \in J$. Hence, Theorem 2 in [27] shows that $V$ is weakly relatively compact in $C$.

As consequence of Claims 1-3 and from Theorem 15, it follows that, for every $w \in \Omega,\{u \in W(w): N(w) u=u\} \neq \emptyset$. Apply now Theorem 16; Steps 1-3 show that, for each $w \in$ $\Omega, N$ has at least one fixed point in $W$. Since $\bigcap_{w \in \Omega}$ int $W(w) \neq$ $\emptyset$, and a measurable selector of int $W$ exists, the operator $N$ has a stochastic fixed point; that is, problem (1) has at least one random solution defined on $[-\xi, a] \times[-\mu, b]$. 


\section{An Example}

Let

$$
E=l^{1}=\left\{w=\left(w_{1}, w_{2}, \ldots, w_{n}, \ldots\right): \sum_{n=1}^{\infty}\left|w_{n}\right|<\infty\right\}
$$

be the Banach space with norm $\|w\|_{E}=\sum_{n=1}^{\infty}\left|w_{n}\right|$ and let $\Omega=$ $(-\infty, 0)$ be equipped with the usual $\sigma$-algebra consisting of Lebesgue measurable subsets of $(-\infty, 0)$. Given a measurable function $u: \Omega \rightarrow C([-7 / 2, e] \times[-5, e])$, consider the functional random integral problem of the following form:

$$
\begin{aligned}
& u(x, y, w) \\
& =\frac{x^{3} e^{-y}}{17+w^{2}} u\left(x-3, y-\frac{4}{3}, w\right) \\
& \quad+\frac{x y^{2}}{10+w^{2}} u(x-2, y-6, w) \\
& \quad+\frac{1}{11+w^{2}+x^{2}+y^{2}} u\left(x-\frac{9}{2}, y-\frac{5}{4}, w\right) \\
& \quad+\frac{w^{2} e^{-x-y-3}}{1+w^{2}+|u(x, y, w)|+\left|{ }^{H} I_{\sigma}^{r} u(x, y, w)\right|}, \\
& \quad(x, y) \in J=[1, e] \times[1, e], w \in \Omega, \\
& u(x, y, w)=\Phi(x, y, w), \quad(x, y) \in \widetilde{J}, w \in \Omega,
\end{aligned}
$$

where $\widetilde{J}:=[-7 / 2, e] \times[-5, e] \backslash(1, e] \times(1, e], m=3$, and $r=\left(r_{1}, r_{2}\right) ; r_{1}, r_{2} \in(0, \infty), u=\left(u_{1}, u_{2}, \ldots, u_{n}, \ldots\right), f=$ $\left(f_{1}, f_{2}, \ldots, f_{n}, \ldots\right)$, and

$$
\begin{aligned}
& f_{n}\left(x, y,{ }^{H} I_{\sigma}^{r} u, u, w\right) \\
& \quad=\frac{w^{2} e^{-x-y-3}}{1+w^{2}+\left|u_{n}(x, y, w)\right|+\left|{ }^{H} I_{\sigma}^{r} u_{n}(x, y, w)\right|} ;
\end{aligned}
$$

$(x, y) \in J=[0,1] \times[0,1], w \in \Omega, n \in \mathbb{N}$, and $\Phi:$ $\widetilde{J} \rightarrow E$ is a continuous and measurable function such that $\phi=\left(\phi_{1}, \phi_{2}, \ldots, \phi_{n}, \ldots\right)$, where

$$
\begin{aligned}
& \phi_{n}(x, 1, w)= \frac{x^{3}}{17+w^{2}} \phi_{n}\left(x-3,-\frac{1}{3}, w\right) \\
&+\frac{1}{11+w^{2}+x^{2}} \phi_{n}\left(x-\frac{9}{2},-\frac{1}{4}, w\right) ; \\
& x \in[0,1], n \in \mathbb{N}, \\
& \phi_{n}(1, y, w)= \frac{1}{11+w^{2}+y^{2}} \phi_{n}\left(-\frac{7}{2}, y-\frac{5}{4}, w\right) ; \\
& y \in[0,1], n \in \mathbb{N} .
\end{aligned}
$$

Set

$$
\begin{aligned}
b_{1}(x, y, w) & =\frac{x^{3} e^{-y}}{17}, \\
b_{2}(x, y) & =\frac{x y^{2}}{10}, \\
b_{3}(x, y, w) & =\frac{1}{11+x^{2}+y^{2}} .
\end{aligned}
$$

Then, $b^{*}=1 / 10$. For each $u, v \in E,(x, y) \in[0,1] \times[0,1]$, and $w \in \Omega$, one has

$$
\|f(x, y, u, v, w)\|_{E} \leq 1+\frac{1}{e^{3}}\left(\|u\|_{E}+\|v\|_{E}\right) .
$$

Hence condition $\left(H_{4}\right)$ is satisfied with $p_{1}^{*}=1$ and $p_{2}^{*}=p_{3}^{*}=$ $1 / e^{3}$.

A simple computation shows that all other conditions of Theorem 18 are satisfied. Consequently, Theorem 18 implies that problem (33) has a random solution defined on $[-7 / 2, e] \times[-5, e]$.

\section{Conflicts of Interest}

The authors declare that there are no conflicts of interest regarding the publication of this paper.

\section{Acknowledgments}

This research is supported by the National Natural Science Foundation of China (11671339).

\section{References}

[1] J. L. Strand, "Random ordinary differential equations," Journal of Differential Equations, vol. 7, pp. 538-553, 1970.

[2] A. T. Bharucha-Reid, "Random algebraic equations," in Probabilistic Methods in Applied MAThematics, Vol. 2, pp. 1-52, Academic Press, New York, 1970.

[3] V. Lupulescu, D. O’Regan, and G. ur Rahman, "Existence results for random fractional differential equations," Opuscula Mathematica, vol. 34, no. 4, pp. 813-825, 2014.

[4] S. Abbas, M. Benchohra, and G. M. N'Guérékata, Topics in Fractional Differential Equations, Springer, New York, NY, USA, 2012.

[5] S. Abbas, M. Benchohra, and G. M. N'Guérékata, Advanced Fractional Differential and Integral Equations, Nova Science Publishers, New York, NY, USA, 2015.

[6] D. Baleanu, K. Diethelm, E. Scalas, and J. J. Trujillo, Fractional Calculus Models and Numerical Methods, vol. 3 of Series on Complexity, Nonlinearity and Chaos, World Scientific, 2012.

[7] A. A. Kilbas, H. M. Srivastava, and J. J. Trujillo, Theory and Applications of Fractional Differential Equations, New York, NY, USA, Elsevier, 2006.

[8] Y. Zhou and L. Peng, "Weak solutions of the time-fractional Navier-Stokes equations and optimal control," Computers \& Mathematics with Applications. An International Journal, vol. 73, no. 6, pp. 1016-1027, 2017.

[9] Y. Zhou and L. Peng, "On the time-fractional Navier-Stokes equations," Computers \& Mathematics with Applications. An International Journal, vol. 73, no. 6, pp. 874-891, 2017.

[10] Y. Zhou, V. Vijayakumar, and R. Murugesu, "Controllability for fractional evolution inclusions without compactness," Evolution Equations and Control Theory, vol. 4, no. 4, pp. 507-524, 2015.

[11] Y. Zhou and L. Zhang, "Existence and multiplicity results of homoclinic solutions for fractional Hamiltonian systems," Computers \& Mathematics with Applications. An International Journal, vol. 73, no. 6, pp. 1325-1345, 2017. 
[12] Y. Zhou, B. Ahmad, and A. Alsaedi, "Existence of nonoscillatory solutions for fractional neutral differential equations," Applied Mathematics Letters, vol. 72, pp. 70-74, 2017.

[13] F. S. De Blasi, "On a property of the unit sphere in a Banach space," vol. 21(69), no. 3-4, pp. 259-262, 1977.

[14] J. Banaś and K. Goebel, Measures of Noncompactness, vol. 60 of Lecture Notes in Pure and Applied Mathematics, Marcel Dekker, New York, NY, USA, 1980.

[15] R. R. Akhmerov, M. I. Kamenskii, A. S. Potapov, A. E. Rodkina, and B. N. Sadovskii, Measures of Noncompactness and Condensing Operators, vol. 55 of Operator Theory: Advances and Applications, Birkhäauser, Basel, Switzerland, 1992.

[16] J. C. Alvàrez, "Measure of noncompactness and fixed points of nonexpansive condensing mappings in locally convex spaces," Real Academia de Ciencias Exactas, Fisicas y Naturales de Madrid. Revista, vol. 79, no. 1-2, pp. 53-66, 1985.

[17] D. Guo, V. Lakshmikantham, and X. Liu, Nonlinear Integral Equations in Abstract Spaces, vol. 373 of Mathematics and its Applications, Kluwer Academic Publishers Group, Dordrecht, The Netherlands, 1996.

[18] D. O'Regan, "Weak solutions of ordinary differential equations in Banach spaces," Applied Mathematics Letters, vol. 12, no. 1, pp. 101-105, 1999.

[19] S. d. Abbas, W. A. Albarakati, M. Benchohra, and J. Henderson, "Existence and Ulam stabilities for Hadamard fractional integral equations with random effects," Electronic Journal of Differential Equations, Paper No. 25, 12 pages, 2016.

[20] B. C. Dhage, S. V. Badgire, and S. K. Ntouyas, "Periodic boundary value problems of second order random differential equations," Electronic Journal of Qualitative Theory of Differential Equations, pp. 1-14, 2009.

[21] B. J. Pettis, "On integration in vector spaces," Transactions of the American Mathematical Society, vol. 44, no. 2, pp. 277-304, 1938.

[22] A. S. Kechris, Classical Descriptive Set Theory, vol. 156 of Graduate Texts in Mathematics, Springer, New York, NY, USA, 1995.

[23] S. Itoh, "Random fixed-point theorems with an application to random differential equations in Banach spaces," Journal of Mathematical Analysis and Applications, vol. 67, no. 2, pp. 261273, 1979.

[24] H. W. Engl, "A general stochastic fixed-point theorem for continuous random operators on stochastic domains," Journal of Mathematical Analysis and Applications, vol. 66, no. 1, pp. 220-231, 1978.

[25] D. O’Regan, "Fixed-point theory for weakly sequentially continuous mappings," Mathematical and Computer Modelling, vol. 27, no. 5, pp. 1-14, 1998.

[26] D. Bugajewski and S. a. Szufla, "Kneser's theorem for weak solutions of the Darboux problem in Banach spaces," Nonlinear Analysis. Theory, Methods \& Applications. An International Multidisciplinary Journal, vol. 20, no. 2, pp. 169-173, 1993.

[27] A. R. Mitchell and C. h. Smith, "Nonlinear Equations in Abstract Spaces," in An existence theorem for weak solutions of differential equations in Banach spaces, V. Lakshmikantham, Ed., pp. 387-403, Academic Press, New York, NY, USA, 1978. 


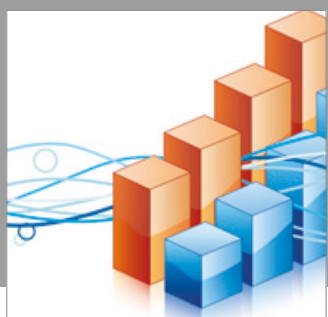

Advances in

Operations Research

vatersals

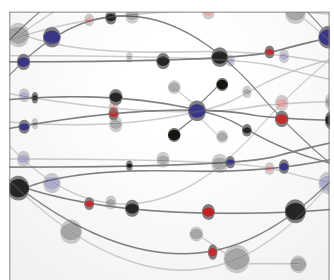

\section{The Scientific} World Journal
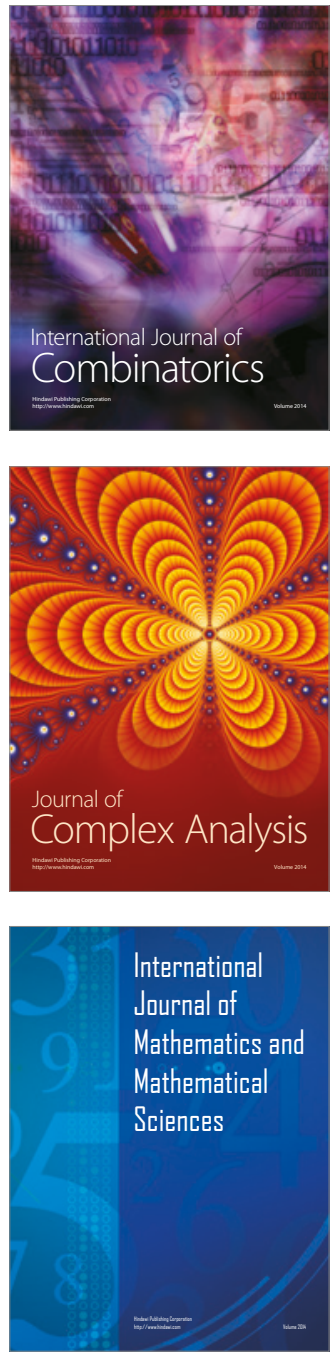
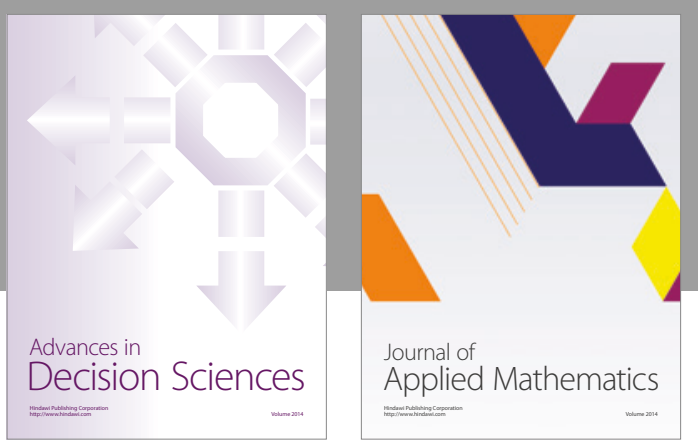

Algebra

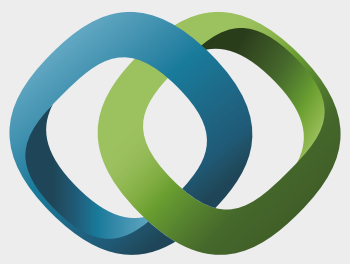

\section{Hindawi}

Submit your manuscripts at

https://www.hindawi.com
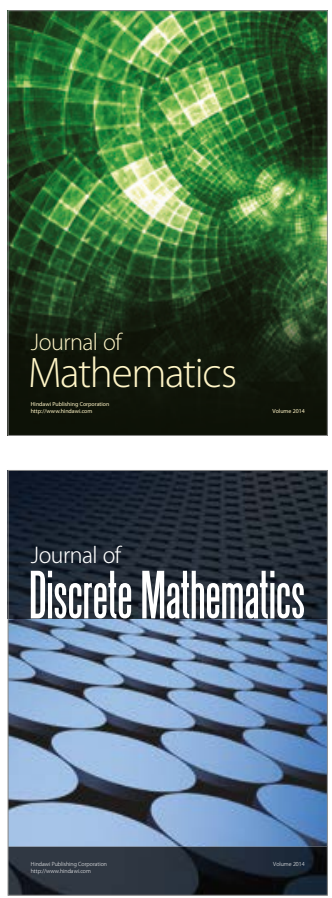

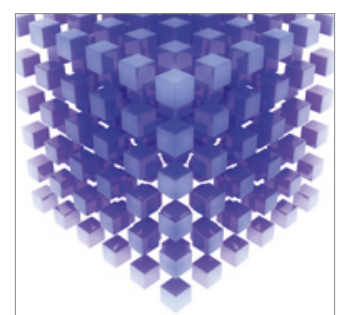

Mathematical Problems in Engineering
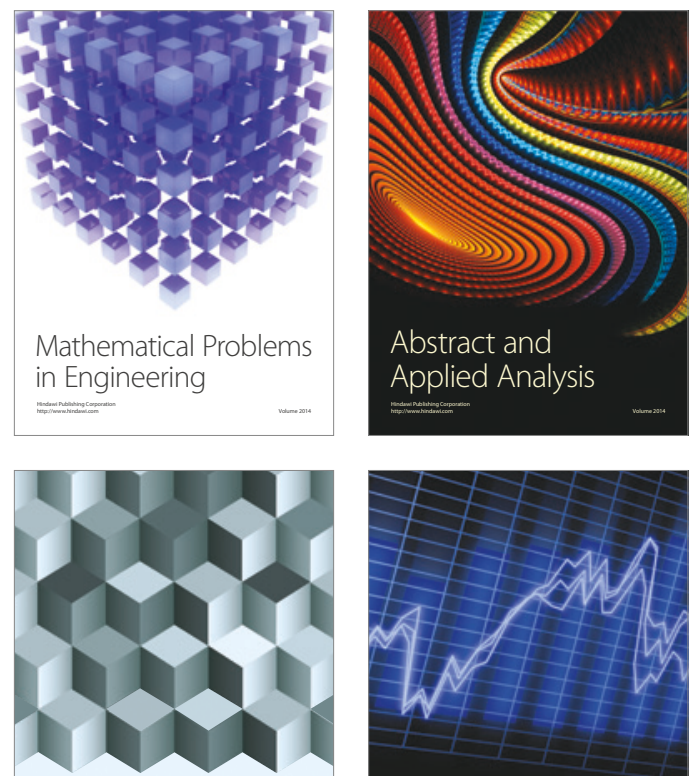

Journal of

Function Spaces

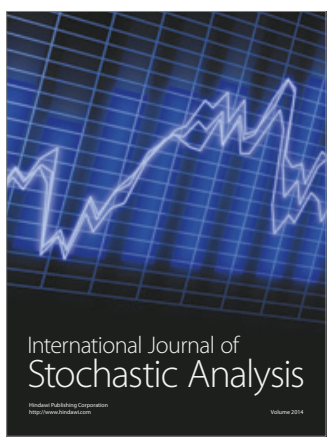

Probability and Statistics
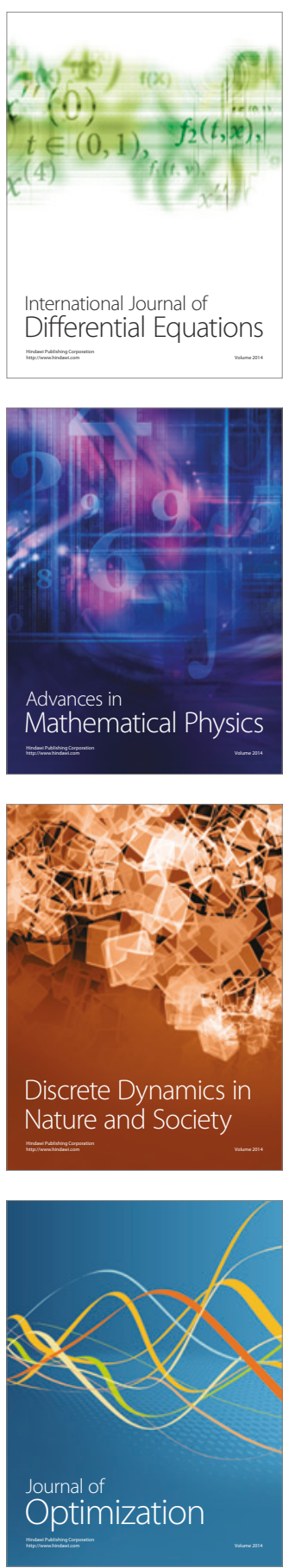